\title{
Merging massive black holes the right place and the right time
}

\author{
Astrid Lamberts \\ Theoretical Astrophysics, California Institute of Technology, \\ Pasadena, CA, 91125, USA \\ email: lamberts@caltech.edu
}

\begin{abstract}
The LIGO/Virgo detections of gravitational waves from merging black holes of $\simeq 30$ solar mass suggest progenitor stars of low metallicity $\left(Z / Z_{\odot} \lesssim 0.3\right)$. In this talk I will provide constrains on where the progenitors of GW150914 and GW170104 may have formed, based on advanced models of galaxy formation and evolution combined with binary population synthesis models. First I will combine estimates of galaxy properties (star-forming gas metallicity, star formation rate and merger rate) across cosmic time to predict the low redshift $\mathrm{BBH}$ merger rate as a function of present day host galaxy mass, formation redshift of the progenitor system and different progenitor metallicities. I will show that the signal is dominated by binaries formed at the peak of star formation in massive galaxies with and binaries formed recently in dwarf galaxies. Then, I will present what very high resolution hydrodynamic simulations of different galaxy types can learn us about their black hole populations.
\end{abstract}

Keywords. galaxies:abundances, stellar content; stars:binaries, black holes, evolution; gravitational waves

\section{Introduction}

The detection of gravitational waves (GW) from merging black holes $(\mathrm{BH})$ and neutron stars has opened a new window on our Universe (LIGO/Virgo (2016), LIGO/Virgo (2017)). More specifically, it has revived the study of massive stars and their binary interactions (Belczynski et al. (2016), Eldridge \& Stanway (2016), Spera \& Mapelli (2017)). While there is a global understanding of binary evolution for field binaries, the exact properties of their mass loss, mass transfer and supernova mechanisms are still unclear (Belczynski et al. (2010), Dominik et al. (2013)). The high masses of the first GW detection, (30 $M_{\odot}$ BHs for GW150914) were unexpected (but see Belczynski et al. (2010)). These high masses suggest low-metallicity progenitors, where mass loss due to winds is reduced. We have limited information on low-metallicity massive stars as massive stars are short-lived and stars recently formed in the Milky Way have a metallicity at least as high as the Sun. In this talk, we determine the conditions where the progenitors of GW150914 may have formed, based on a semi-analytic formalism of galaxy evolution and a binary population synthesis model. We determine the formation rate of GW150914like progenitors based on the mass of the host galaxy and the formation time of the progenitor. We refer the reader to Lamberts et al. (2016) for a more detailed description of the method. We then present a preliminary analysis of the merger rates predicted by cosmological simulations.

\section{Forming low-metallicity stars}

The first step is to determine the conditions for low-metallicity star formation in the universe. Globally, the metallicity in the Universe increases as successive generations of 

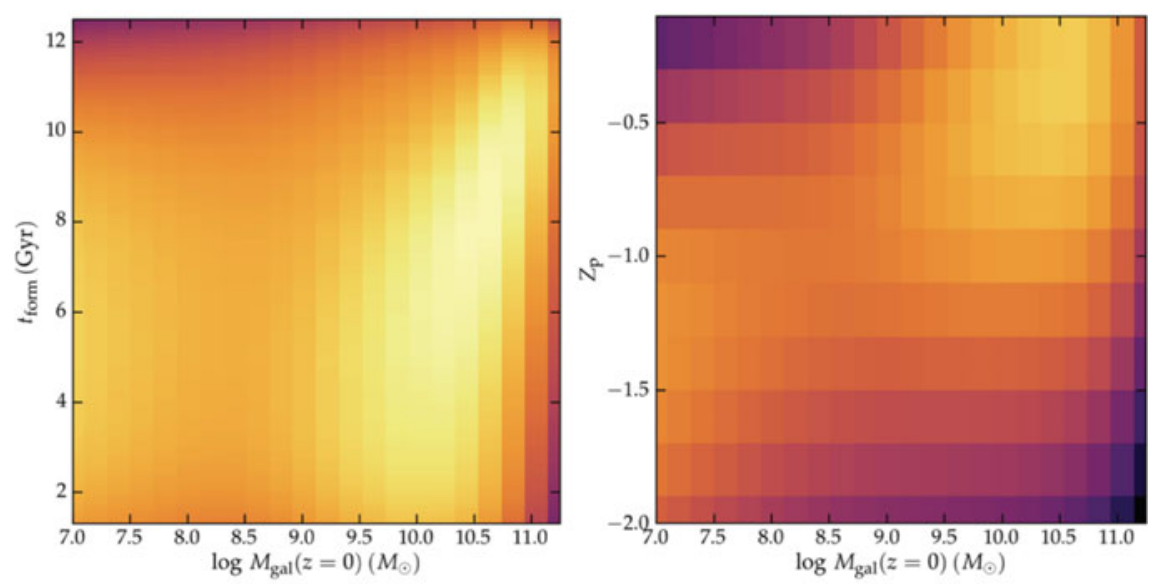

Figure 1. Low-metallicity star formation rate (arbitrary units) as a function of galaxy mass and lookback time to formation (left) and metallicity (right).

stars progressively enrich the star-forming gas. At a given epoch galaxies follow the massmetallicity relation (Kewley \& Ellison (2008)), with more massive galaxies being more metal rich. For each present-day galaxy mass, we determine the mass of the corresponding dark matter halo using abundance matching by Behroozi et al. (2013). Based on the model by Behroozi et al. (2013) we then determine the amount of star formation in each halo in 10 Myr time bins. Based on the redshift-dependent mass-metallicity relation computed by Ma et al. (2016), we derive the stellar mass formed in 11 metallicity bins between $Z=0.01 Z_{\odot}$ and $Z=1 Z_{\odot}$, where $Z_{\odot} \equiv 0.02$. We include scatter between different galaxies of $\sigma=0.1 \mathrm{dex}$, based on observations by Tremonti et al. (2004). We also include $\sigma=0.2$ scatter of the metallicity within galaxies, according to Berg et al. (2013). Fig. 1 shows the normalized star formation rate as a function of present-day galaxy mass and lookback time to formation (left) and metallicity (right). The figure only shows subsolar metallicity star-formation, which is relevant to binary BH formation. However, particularly in massive galaxies, a large fraction of the stars form at higher metallicities. We find a slightly bimodal distribution, with most of the low-metallicity stars forming in massive (Milky-Way like) galaxies around the peak of cosmic star-formation (between 6 and 10 Gyrs ago). More recent low-metallicity star formation also occurs in dwarf galaxies, typically at metallicity below 10 per cent of Solar. In the next section, we will highlight how metallicity affects binary $\mathrm{BH}$ formation and merger rates and show which low-metallicity stars shown in Fig. 1 mostly contribute to GW150914-like events observed now.

\section{Forming binary black hole merger progenitors}

We use the BSE code (Hurley et al. (2002)) to compute the delay time distribution of binary $\mathrm{BH}$ mergers with respect to the formation of the stellar progenitor. We neglect BH mergers from globular clusters (Rodriguez et al. (2015)) and consider a single set of standard assumptions on binary evolution. We have updated BSE to account for improvement models of stellar winds (Belczynski et al. (2010)), remnant masses (Belczynski et al. (2008)) and BH kicks due to supernova explosions (Dominik et al. (2013)). Binaries undergoing common-enveloppe mass-transfer during the Hertzprung gap merge as stars. At later stages, we set the common enveloppe efficiency to unity and assume that during Roche lobe overflow, only half of the mass is accreted by the companion. 


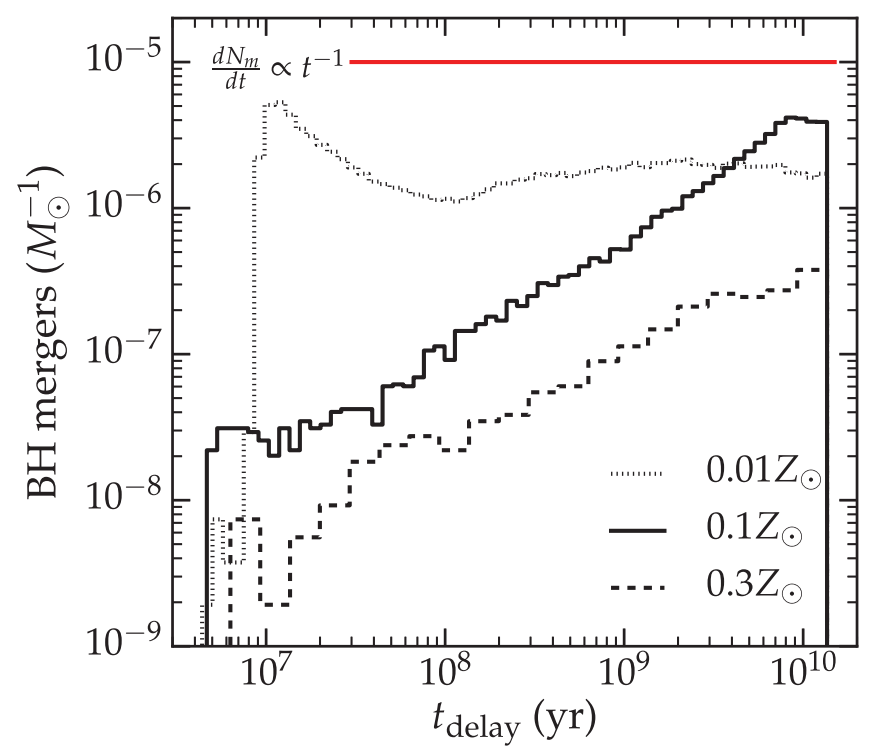

Figure 2. Delay time distribution for massive binary black hole mergers with progenitors of different metallicities. Taken from Lamberts et al. (2016).

For each metallicity bin, we model $2.5 \times 10^{6}$ binaries with primary masses between 25 and $150 M_{\odot}$ following the Kroupa (2001) initial mass-function. The initial period distributions and mass ratios are derived from observations by Sana et al. (2012) and we use thermal distribution for the initial eccentricity. Fig. 2 shows the BH mergers with total mass above $40 M_{\odot}$ (per unit solar mass) for $Z=0.01,0.1,0.3 Z_{\odot}$ according to our models. The lowest metallicity stars produce the most massive $\mathrm{BH}$, as their mass-loss is limited and early mergers. At later times, more metal-rich stars contribute more. Stars with metallicity above $0.3 \mathrm{Z}_{\odot}$ produce 10 times less mergers of these high masses, owing to the strong mass loss of the progenitor stars.

We combine our semi-analytic model for low-metallicity star-formation (Fig. 1) and the metallicity-dependent delay-time distribution (Fig. 2) to determine where and when the progenitor of GW150914 most likely formed. For all stars formed, at all timesteps, we count the resulting $\mathrm{BH}$ mergers occurring between $z=0.1$ and the present day. The conditions of formation of the progenitors are shown in Fig. 3. The figure has the same axes as Fig. 1 and the colors can be directly compared. It shows that the bimodality in lowmetallicity star formation is enhanced. Roughly half of the progenitors of GW150914-like systems stem from Milky-Way like galaxies $\left(M \simeq 10^{11} M_{\odot}\right)$ and were formed 6 to 10 Gyrs ago, at roughly 10 per cent of Solar metallicity. Later star formation in massive galaxies has too high metallicity to significantly contribute to $\mathrm{BH}$ merger progenitors. Recent star formation in dwarf galaxies also significantly contributes to $\mathrm{BH}$ mergers, stemming from progenitors with metallicities below 10 per cent of Solar. Because of their low-metallicity, galaxies below $10^{8} M_{\odot}$ overproduce $\mathrm{BH}$ mergers in comparison with their contribution to the global star formation. These galaxies are faint, and unobservable at high redshift and $\mathrm{BH}$ mergers may be the only way to infer some of their properties. The side panel of the left figure shows the formation time of the progenitors of massive $\mathrm{BH}$ mergers, which is mostly uniform over the past 7 Gyrs. This differs from the bimodal distribution from Belczynski et al. (2016), because of our improved model of low-metallicity star-formation. In the next section we present preliminary results from cosmological simulations. 

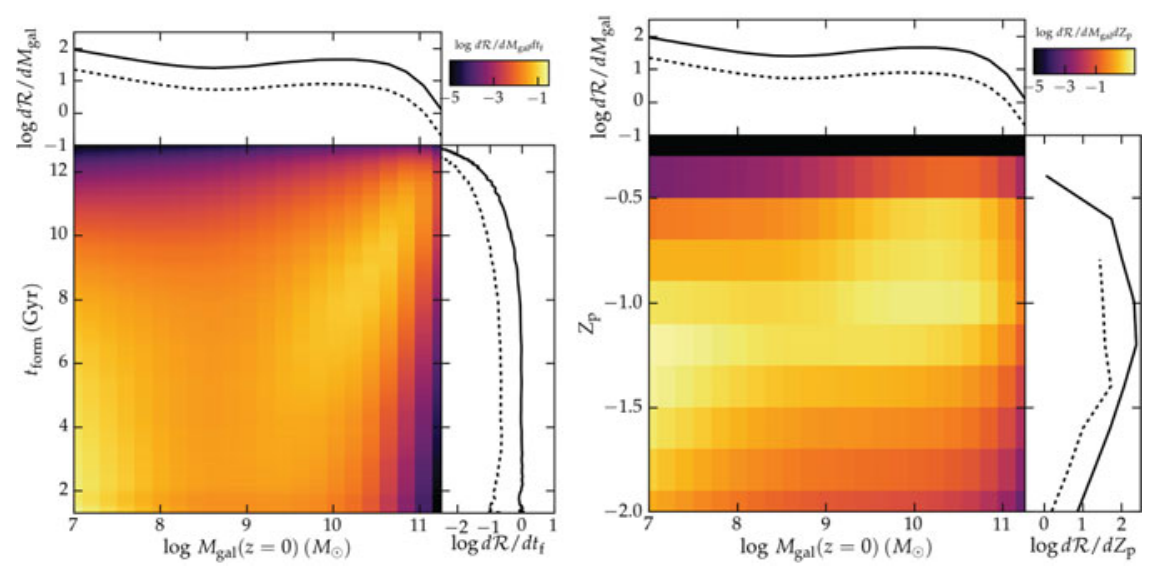

Figure 3. Formation rate of progenitors of massive black hole mergers as a function of galaxy mass and lookback time to formation (left) and metallicity (right). Taken from Lamberts et al. (2016).

\section{Preliminary results from high-resolution simulations}

We have performed a comparable analysis based on hydrodynamical simulations of different galaxies. We use simulations from the Feedback In Realistic Environments project (FIRE ,Hopkins et al. (2014)). The cosmological simulations are ran with the GIZMO code (Hopkins (2013)) and include a multiphase model of the interstellar medium and stellar feedback based on a complete stellar evolution model. We specifically use the "Latte" simulation (Wetzel et al. (2016)), a cosmological simulation of a Milky-Way like galaxy with a mass resolution of $7070 M_{\odot}$. The left panel of fig. 4 shows the normalized star-formation rate in a Milky-Way like galaxy. The right panel only shows the stars contributing to $\mathrm{BH}$ mergers within the detector horizon during the second observing run. We have directly associated the delay time distributions (Fig. 2) with the star formation in the simulation. We find that mergers come from low-metallicity star-formation and that effectively most of the stars formed in Milky-Way-like galaxies are unable to form merging black holes. As in Lamberts et al. (2016), we find that the mergers come from star formation between 6 and 10 Gyrs ago. Fig. 5 shows the total number of mergers as a function of time in different metallicity bins. Again, we confirm the findings from Lamberts et al. (2016), with the largest contribution coming from $Z=0.03-0.13 Z_{\odot}$. We find that the number of mergers increases towards redshift $z=2$ (10Gyrs ago), somewhat following the global star formation history of the galaxy. As the horizon of the detectors increases, we thus expected an increase of the number of detections per unit volume for contributions from massive galaxies.

\section{Implications}

In this talk, we presented a unique combination between a binary population synthesis model and a complete semi-analytic cosmological model for low-metallicity star formation. We derive the contribution of different galaxies to the present-day merger rate of massive $\left(>30 M_{\odot}\right)$ binary black holes. We find that the progenitors of such mergers could have formed during the peak of star formation (between 6 and 10 Gys argo) in a galaxy that would now resemble the Milky Way or more recently $(<6$ Gyrs ago) in a dwarf galaxy. Our method solely relies on the strong metallicity dependence of binary black hole formation (and mergers) to determine the relative contributions of different galaxies to 

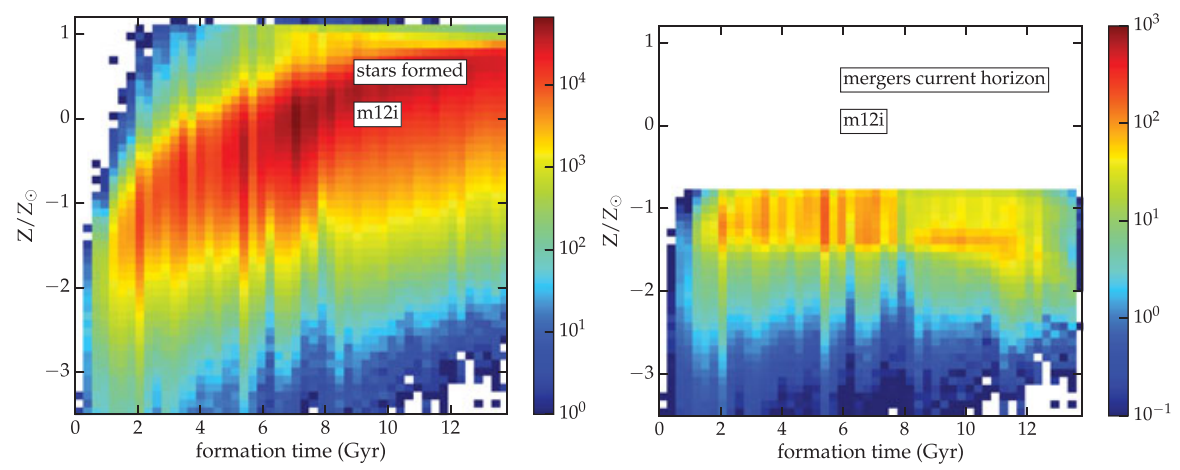

Figure 4. Normalized star formation rate (left) and formation rate of BBH merger progenitors (right) from the Latte simulation.

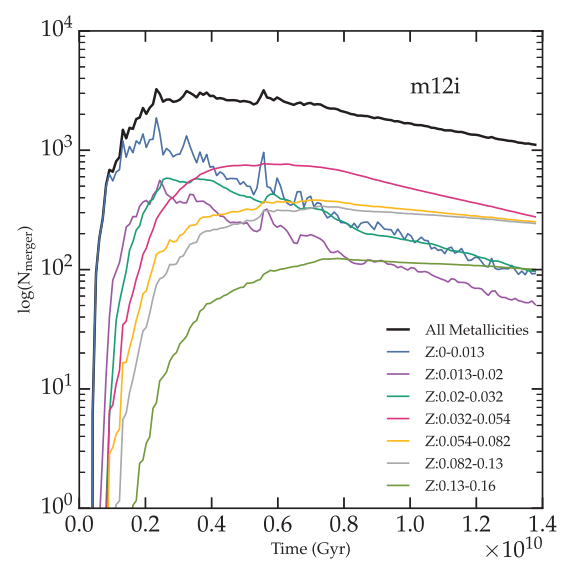

Figure 5. Number of mergers in a Milky-Way like galaxy as a function of time for different metallicities of the progenitor stars. The total number of mergers is shown in the thick black line.

present-day black hole mergers. Although absolute merger rate we predict is in agreement with the LIGO/Virgo detection rate (LIGO/Virgo (2016)), we emphasize that it may be revised as our understanding of massive binary evolution improves. We confirm our findings using the same binary evolution model combined with a high-resolution simulation of a Milky-way like galaxy. We find that the merger rate in massive galaxies increases towards redshift $z=2$ and hundreds of detections are expected when LIGO and Virgo reach their design sensitivity (about $z \simeq 1$ ). As such, these detections will provide strong constrains on binary evolution models and star formation in high-redshift and/or faint galaxies.

\section{References}

Behroozi, P. S., Wechsler, R. H., \& Conroy, C. 2013 ApJ, 770, 57

Belczynski, K., Holz, D. E., Bulik, T. \& O'Shaughnessy, R. 2016 Nature, 734, 512

Belczynski, K., Bulik, T., Fryer, C. L., Ruiter, A., Valsecchi, F., Vink, J. S., \& Hurley, J. R. 2010 ApJ, 714, 1217

Belczynski, K., Dominik, M., Bulik, T., O'Shaughnessy, R. Fryer, C., \& Holz, D. E. 2010 ApJL, 715, 138 
Belczynski, K., Kalogera, V., Rasio, F. A., Taam, R. E., Zezas, A., Bulik, T., Maccarone, T. J., \& Ivanova, N. 2008 ApJ, 174, 223

Berg, D. A., Skillman, E. D., Garnett, D. R., Croxall, K. V., Marble, A. R., Smith, J. D., Gordon, K., \& Kennicutt, Jr., R. C., 2013 ApJ, 775, 128

Dominik, M., Belczynski, K., Fryer, C., Holz, D. E., Berti, E., Bulik, T., Mandel, I., \& O'Shaughnessy, R. 2013 ApJ, 759,52

Eldridge, J. J. \& Stanway, E. R. 2016 MNRAS, 462, 3302

Hopkins, P. F. 2013 MNRAS, 428, 2840

Hopkins, P. F., Kereš, D., Oñorbe, J., Faucher-Giguère, C.-A., Quataert, E., Murray, N., \& Bullock, J. S. $2014 M N R A S, 445,581$

Hurley, J. R., Tout, C. A., \& Pols, O.R. MNRAS, 329, 897

Kewley, L. J. \& Elisson, S. E. 2008 ApJ, 681, 1183

Kroupa, P. 2001 MNRAS, 322, 231

Lamberts, A., Garrison-Kimmel, S., Clausen, D., \& Hopkins, P. F. . 2016 MNRASL, 463, 31

LIGO/Virgo Scientific Collaboration 2016 ApJL, 833, 1

LIGO/Virgo Scientific Collaboration 2016 PRL, 116, 061102

LIGO/Virgo Scientific Collaboration 2017 PRL, 119, 161101

Ma, X., Hopkins, P. F., Faucher-Giguère, C.-A., Zolman, N., Muratov, A. L., Kereš, D., \& Quataert, E. 2016 MNRAS, 456, 2140

Rodriguez, C. L., Morscher, M., Pattabiraman, B., Chatterjee, S., Haster, C.-J., \& Rasio, F. A. 2017 PRL, 5, 051101

Sana, H., de Mink, S. E., de Koter, A., Langer, N., Evans, C. J., Gieles, M., Gosset, E., Izzard, R. G., Le Bouquin, J.-B., \& Schneider, F. R. N 2012 Science, 337, 444

Spera, M. \& Mapelli, M. 2017 MNRAS, 470, 4739

Tremonti, C. A., Heckman, T. M., Kauffmann, G.\& Brinchmann, J., Charlot, S., White, S. D. M., Seibert, M., Peng, E. W., Schlegel, D. J., Uomoto, A., Fukugita, M., \& Brinkmann, J. 2004 ApJ, 613, 898

Wetzel, A. R., Hopkins, P. F., Kim, J.-h., Faucher-Giguère, C.-A., Kereš, D., \& Quataert, E. 2016 ApjL, 827, L23 